\title{
Analytical solution of the Bohr-Mottelson equation in minimal length effect for cotangent hyperbolic potential using the hypergeometric method
}

\author{
Siti Noor Fatimah ${ }^{1}$, A Suparmi ${ }^{2}$, C Cari ${ }^{3}$, and Isnaini Lilis Elviyanti ${ }^{4}$ \\ ${ }^{1,2,3,4}$ Physics Department, Graduate Program, Sebelas Maret University, \\ Jl. Ir. Sutami No. 36 Kentingan Surakarta \\ E-mail: ${ }^{1}$ fatmalida@gmail.com, $*$ soeparmi@ staff.uns.ac.id
}

Received 13 February 2018, Revised 25 February 2018, Published 31 March 2018

\begin{abstract}
The rigid deformed nucleus of minimal length effect is investigated using the Bohr-Mottelson equation that influenced by cotangent hyperbolic potential. The Bohr-Mottelson equation in effect a minimum length resolved hypergeometric method for determining the energy spectrum and the wave functions. Energy spectrum was calculated using Matlab software and the wave function is displayed in the form of hypergeometric.
\end{abstract}

Keywords: Bohr Mottelson, minimum length, hypergeometric

\section{Introduction}

The Bohr-Mottelson equation is used to explain the behavior of nucleus such as the rigid deformed nucleus (Alimohammadi et al.: 2017; Chabab et al.: 2015; Chabab et al.: 2016). The rigid deformed nucleus occur nucleus rotation motion at low excitation energy (Alimohammadi et al.: 2017; Chabab et al.: 2015; Chabab et al.: 2016). The Bohr-Mottelson for various potential has been solved using Super Symmetric Quantum (SUSY QM) for Davidson potential (Bonatsos et al., 2011), Nikiforov-Uvarov for Eckart potential (Naderia et al., 2016) and Asymptotic Iteration Method (AIM) for Hulthen and Ring Shape potential (Chabab et al., 2015).

The concept of minimal length is connected by commutation relations between position and momentum operators in Heisenberg Uncertainty Principle. The minimal length occur at Heisenberg Uncertainty Principle is influenced by gravity quantum which is called General Uncertainty Principle (GUP) (Alimohammadi et al:: 2017; Chabab et al.: 2015; Chabab et al.: 2016; Hossenfelder.: 2004; Garay.: 1994). The General Uncertainty Principle modify Heisenberg Uncertainty Principle with additional a small constant (Chabab et al., 2015).

In this paper, equation Bohr-Mottelson in length at least to the potential effects of Hyperbolic cotangent solved using hypergeometric method. Energy spectrum and the wave functions obtained using methods hypergeometric. Hyperbolic cotangent potentially be used to describe the core excitation (Cari et al.: 2013; Suparmi et al.: 2017). This paper consists of four parts, the second part describes the Bohr-Mottelson 
equation in effect a minimum length and hypergeometric method. Furthermore, in Section 3 describes the results and discussion and final section 4 contains conclusions.

\section{Research Methods}

\subsection{The Bohr-Mottelson equation in minimal length effect}

The general canonical commutation between position and momentum is expressed (Hossenfelder.: 2004; Garay.: 1994)

$$
[X, P] \geq i \hbar
$$

where $X$ is a position, $P$ is a corresponding momentum. Then, the general canonical commutation between position and momentum is influenced by quantum gravity, it is becomes (Alimohammadi et al.: 2017; Chabab et al.: 2015; Chabab et al.: 2016; Hossenfelder.: 2004; Garay.: 1994),

$$
[X, P] \geq i \hbar\left(1+\alpha(\Delta P)^{2}\right)
$$

The equation (2) is called General Uncertainty Principle, where $\alpha$ was a minimal length parameter that has very small positive values. The uncertainty relation is caused by commutation relation. The equation (2) can be reduced becomes (Alimohammadi et al.: 2017; Chabab et al.: 2015; Chabab et al.: 2016; Hossenfelder.: 2004; Garay.: 1994)

$$
\begin{gathered}
\hat{X}_{i}=\hat{x}_{i} \\
\hat{P}_{i}=\left(1+\alpha \hat{p}^{2}\right) \hat{p}_{i}
\end{gathered}
$$

Then, equation (4) can be written (Alimohammadi et al.: 2017; Chabab et al.: 2016)

$$
P^{2}=-\frac{\hbar^{2}}{2 B_{m}}(1-2 \alpha \Delta) \Delta
$$

where $\Delta$ is Laplacian operator for nucleus that has three degrees of freedom : $q_{1}=\phi, q_{2}=\theta, q_{3}=\beta$, the Laplacian operator as follow (Alimohammadi et al:: 2017; Chabab et al.: 2016)

$$
\Delta=\frac{1}{\sqrt{g}} \sum_{i, j} \frac{\partial}{\partial q_{i}} \sqrt{g} g_{i j}^{-1} \frac{\partial}{\partial q_{j}}
$$

with $g$ and $g_{i j}^{-1}$ are determinant and inverse of the matrix $g_{i j}$, respectively. We get Laplacian operator, is given as (Alimohammadi et al:: 2017; Chabab et al.: 2016)

$$
\Delta=\left[\frac{1}{\beta^{2}} \frac{\partial}{\partial \beta} \beta^{2} \frac{\partial}{\partial \beta}+\frac{1}{3 \beta^{2}}\left[\frac{1}{\sin \theta} \frac{\partial}{\partial \theta} \sin \theta \frac{\partial}{\partial \theta}+\frac{1}{\sin ^{2} \theta} \frac{\partial^{2}}{\partial \varphi^{2}}\right]\right]
$$

Then equation (5) is inserted in Hamiltonian equation which is expressed,

$$
H=T+V(\beta)=\frac{P^{2}}{2 B_{m}}+V(\beta)
$$

where $P$ is momentum operator, $V(\beta)$ is potential energy in $\beta$ function and $B_{m}$ is a mass parameter. We obtain, 


$$
\left[-\frac{\hbar^{2}}{2 B_{m}} \Delta+\frac{\alpha \hbar^{4}}{B_{m}} \Delta^{2}+V(\beta, \theta, \phi)-E\right] \Psi(\beta, \theta, \phi)=0
$$

The equation (9) is Bohr-Mottelson equation in minimal length effect. In the case of Bohr-Mottelson equation without the minimal length effect with $\alpha_{M L}=0$ (Elviyanti et al.: 2017) for equation (9), so yields square term is given as (Alimohammadi et al:: 2017),

$$
\Delta^{2}=\frac{4 B_{m}^{2}}{\hbar^{4}}\left(V(\beta)-E^{0}\right)^{2}
$$

Equations (7) and (10) are inserted in equation (9) and multiplied by $-\frac{2 B_{m}}{\hbar^{2}}$ and $\hbar=1$ (natural unit), is yields

$$
\left[\left[\begin{array}{l}
\frac{1}{\beta^{2}} \frac{\partial}{\partial \beta} \beta^{2} \frac{\partial}{\partial \beta}+ \\
\frac{1}{3 \beta^{2}}\left(\begin{array}{l}
\frac{1}{\sin \theta} \frac{\partial}{\partial \theta} \sin \theta \frac{\partial}{\partial \theta} \\
+\frac{1}{\sin ^{2} \theta} \frac{\partial^{2}}{\partial \varphi^{2}}
\end{array}\right)
\end{array}\right]+2 B_{m}\left(\begin{array}{l}
4 \alpha_{M L} B_{m}\left(V(\beta)-E^{0}\right)^{2} \\
+(V(\beta)-E)
\end{array}\right] \Psi(\beta, \theta, \varphi)=0\right.
$$

By setting $\Psi(\beta, \theta, \varphi)=R(\beta) \Theta(\theta) \Phi(\varphi)$ which is the separation variable method that used to solve equation (11), we have Euler angles part of Bohr-Mottelson Hamiltonian with minimal length,

$$
-\left(\frac{1}{\Phi(\varphi)} \frac{1}{\sin ^{2} \theta} \frac{\partial^{2} \Phi(\varphi)}{\partial \varphi^{2}}+\frac{1}{\Theta(\theta)} \frac{1}{\sin \theta} \frac{\partial}{\partial \theta} \sin \theta \frac{\partial \Theta(\theta)}{\partial \theta}\right)=\lambda
$$

and $\beta-$ part of Bohr-Mottelson Hamiltonian with minimal length,

$$
\left\{\begin{array}{l}
\frac{1}{\beta^{2}} \frac{\partial}{\partial \beta} \beta^{2} \frac{\partial R(\beta)}{\partial \beta}+2 B_{m}(E-V(\beta)) R(\beta) \\
-8 B_{m}{ }^{2} \alpha_{M L}\left(E_{0}^{2}-2 E_{0} V(\beta)+V^{2}(\beta)\right) R(\beta)
\end{array}\right\}=\frac{\lambda}{3 \beta^{2}} R(\beta)
$$

By applying $R(\beta)=F(\beta) / \beta$ and $\lambda=L(L+1)$ in equation (13) so we have,

$$
\left\{\begin{array}{l}
\frac{d^{2} F(\beta)}{d \beta^{2}}-\frac{L(L+1)}{3 \beta^{2}} F(\beta) \\
+2 B_{m}(E-V(\beta)) F(\beta) \\
-8 B_{m}{ }^{2} \alpha_{M L}\left(E^{2}-2 E V(\beta)+V^{2}(\beta)\right) F(\beta)
\end{array}\right\}=0
$$

The Bohr-Mottelson equation for a $\beta$-part in a minimal length effect for rigid deformed nucleus case is expressed by equation. 


\subsection{Hypergeometric method}

The second-order differential equation of hypergeometric function as follow (Suparmi.: 2011; Elviyanti et al.: 2017),

$$
y(1-y) \frac{d^{2} \Phi}{d y^{2}}+(c-(a+b+1) y) \frac{d \Phi}{d y}-a b \Phi=0
$$

The energy eigenvalue is obtained from the condition in equation (15), (Suparmi.: 2011; Elviyanti et al.: 2017)

$$
a=-n \text { or } b=-n
$$

where $n=0,1,2,3 \ldots$ Equation (16) can be finite series of polynomials of rank $n$ by equation (15). The solution of a wave function is given as

$$
\Phi(y)={ }_{2} F_{1}(a, b, c, y)=\sum_{m=0} \frac{(a)_{m}(b)_{m}}{(c)_{m} n !} y^{m}=1+\frac{a b}{c} y+\frac{a(a+1) b(b+1)}{c(c+1)} \frac{y^{2}}{2 !}+\ldots
$$

By applying the suitable variable change in equation and reduced to standard hypergeometri equation, we get energy eigenvalue and wave function (Suparmi.: 2011; Elviyanti et al.: 2017)

\section{Result and discussion}

Cotangent hyperbolic potential is expressed as follows:

$$
V(\beta)=V_{o} \operatorname{coth} \eta \beta+V_{1}
$$

with $V_{0}$ and $V_{l}$ is a constant potential, and $\eta$ is a potential difference. Substituting equation (18) into the equation (14) it is obtained

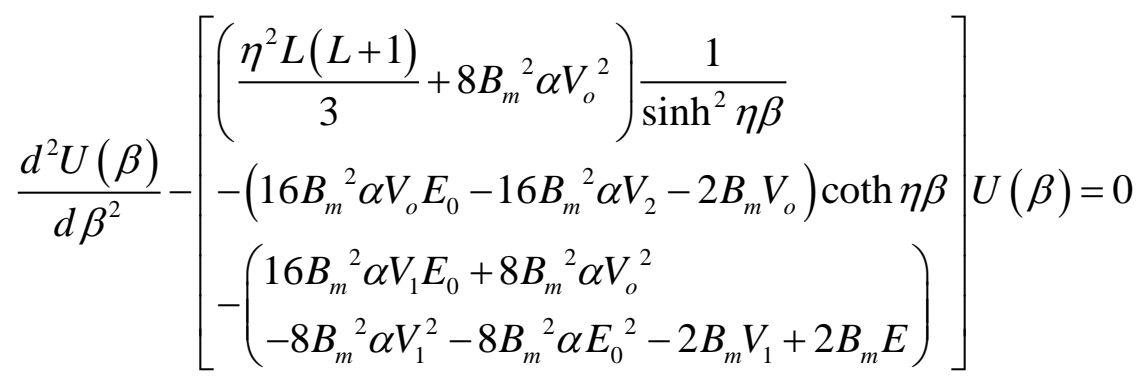

by setting,

$$
\begin{gathered}
v(v-1)=\left(\frac{\eta^{2} L(L+1)}{3}+8 B_{m}{ }^{2} \alpha V_{o}{ }^{2}\right) \\
2 q=\left(16 B_{m}{ }^{2} \alpha V_{o} E_{0}-16 B_{m}{ }^{2} \alpha V_{2}-2 B_{m} V_{o}\right) \\
-k^{2}=\left(\begin{array}{l}
16 B_{m}{ }^{2} \alpha V_{1} E_{0}+8 B_{m}{ }^{2} \alpha V_{o}{ }^{2} \\
-8 B_{m}{ }^{2} \alpha V_{1}{ }^{2}-8 B_{m}{ }^{2} \alpha E_{0}{ }^{2}-2 B_{m} V_{1}+2 B_{m} E
\end{array}\right)
\end{gathered}
$$

Equation (19) reduces to

$$
\frac{d^{2} U(\beta)}{d \beta^{2}}-\left[\frac{v(v-1)}{\sinh ^{2} \eta \beta}-2 q \operatorname{coth} \eta \beta+k^{2}\right] U(\beta)=0
$$


Equation (23) is a differential equation that has been simplified to the form using the hypergeometric differential equation $\operatorname{coth}(\eta \beta)=(1-2 y)$, We get

$$
y(1-y) \frac{d^{2} U(\beta)}{d y^{2}}+(1-2 y) \frac{d U(\beta)}{d y}+\left[v^{\prime}\left(v^{\prime}-1\right)-\frac{4 \alpha_{H}^{2}}{4 y}-\frac{4 \beta_{H}^{2}}{4(1-y)}\right] U(\beta)=0
$$

with

$$
\frac{-2 q+k^{2}}{\eta^{2}}=4 \alpha_{H}^{2} \quad \frac{2 q+\kappa^{2}}{\eta^{2}}=4 \beta_{H}^{2} \quad v^{\prime}\left(v^{\prime}-1\right)=\frac{v(v-1)}{\eta^{2}}
$$

by inserting the following new wave function

$$
U(\beta)=y^{\alpha_{H}}(1-y)^{\beta_{H}} f(y)
$$

in equation (24) we get

$$
\left[\begin{array}{l}
y(1-y) \frac{f^{2}(y)}{d y^{2}}+\left[\left(2 \alpha_{H}+1\right)-\left(2 \alpha_{H}+2 \beta_{H}+2\right) y\right] \frac{f(y)}{d y} \\
+\left[v^{\prime}\left(v^{\prime}-1\right)-\left(\alpha_{H}+\beta_{H}\right)\left(\alpha_{H}+\beta_{H}+1\right)\right] f(y)
\end{array}\right]=0
$$

Equation (27) is the hypergeometric differential equations are obtained following hypergeometric parameter2

$$
a=\alpha_{H}+\beta_{H}+v^{\prime}-1, b=\alpha_{H}+\beta_{H}+v^{\prime}, c=2 \alpha_{H}+1
$$

By inserting the equation (20) - (22), (25) and (28), we get

$$
\left.E=\frac{\eta^{2}}{2 B_{m}}\left[-\left[\begin{array}{l}
\left(\sqrt{\left(\frac{L(L+1)}{3}+\frac{8 B_{m}{ }^{2} \alpha V_{o}^{2}}{\eta^{2}}+\frac{1}{4}\right)}+\frac{1}{2}-n\right)^{2} \\
\eta^{2}
\end{array}\right]\right)^{2}\right]+\left[\begin{array}{l}
4 B_{m} \alpha V_{1}^{2}+4 B_{m} \alpha E_{0}^{2} \\
+V_{1}-8 B_{m} \alpha V_{1} E_{0}-4 B_{m} \alpha V_{o}{ }^{2}
\end{array}\right]
$$

Equation (29) is the equation of the energy spectrum of the Bohr-Mottelson in length at least to the potential effects of Hyperbolic cotangent. Then, to get the wave function using equation (17), (26) and $\operatorname{coth}(\eta \beta)=(1-2 y)$, We get

$$
U(\beta)=\frac{(1-\operatorname{coth}(\eta \beta))^{\alpha_{H}}}{2} \frac{(1+\operatorname{coth}(\eta \beta))^{\beta_{H}}}{2}{ }_{2} F_{1}(a, b, c, z)
$$

and by inserting equation (28) into the equation (30), we obtain

$$
U(\beta)=\frac{(1-\operatorname{coth}(\eta \beta))^{\alpha_{H}}}{2} \frac{(1+\operatorname{coth}(\eta \beta))^{\beta_{H}}}{2}
$$




$$
U(\beta)=\frac{(1-\operatorname{coth}(\eta \beta))^{\alpha_{H}}}{2} \frac{(1+\operatorname{coth}(\eta \beta))^{\beta_{H}}}{2}\left[1+\frac{(-n)\left(\alpha_{H}+\beta_{H}+v-1\right)\left(\frac{1-\operatorname{coth}(\eta \beta)}{2}\right)}{\left(2 \alpha_{H}+1\right)}\right]
$$

Equation (31) and (32) is a function of the wave equation Bohr-Mottelson in effect a minimum length for $n=0$ and $n=1$. The value of the wave function depends on the value of the parameter hypergeomtric.

\section{Conclusion}

The Bohr-Mottelson equation in minimal length at least to the potential effects of cotangent hyperbolic can be solved by using hypergeometric. Hypergeometric method used to obtain the energy spectrum and the wave functions in the equation BohrMottelson in effect a minimal length. Energy spectrum and the wave functions equation Bohr-Mottelson the minimal length effect can be shown by equation (29), (31) and equation (32).

\section{Acknowledgement}

This research was partly supported by Ministry of Research, Technology and Higher Education with contract no. 089/SP2H/LT/DRPM/2018.

\section{References}

Alimohammadi, M., \& Hassanabadi, H. (2017). Harmonic alternatif solution of the gamma-rigid Bohr Hamiltonian in minimal length formalism. Nuclear Physic A, 957, 439-449

Bonatsos, D., Georgoudis, P.E., Lenis, D., Minkov, N \& Quesne, C. (2011). Bohr Hamiltonian with a deformation - dependent mass term for the Davidson potential. Physical Review C, 83,044321

Cari, C., Suparmi, A., Deta, U.A., \& Werdiningsih, I.S. (2013). Solution of Dirac Equations for Cotangent Potential with Coulomb-type Tensor Interaction for Spin and Pseudospin Symmetries Using Romanovski Polynomials. Makara J. Sci, 93-102

Chabab, M., Lahbas, A., \& Oulne, M. (2015). Bohr Mottelson with Hulthen plus ringshaped potential for triaxial nuclei. The European physical Journal A, 51, 13101-13113

Chabab, M., El Batoul, A., Lahbas, A., \& Oulne, M. (2016). On $\gamma$ - rigid regime of the Bohr Mottelson Hamiltonian in the presence of a minimal length. Physics Letters $B, 758,212-218$

Elviyanti, I.L., Suparmi, A., Cari,C., Nugraha, D,A., \& Pratiwi, B, N. (2017). Solution of Klein Gordon equation for hyperbolic cotangent potential in the presence of a minimal length using Hypergeometric method. IOP Conf. Series : Journal of Physics: Conf. Series 909012023

Garay, L.J. (1994). Quantum gravity and minimum length. International Journal of 
Modern Physics A,10, 145-165

Hossenfelder, S. (2004). The Minimal Length And Large Extra Dimensions. Modern Physics Letters A, 19, 37, 2727-2744

Naderia, L \& Hassanabadi, H. (2016). Bohr Hamiltonian with Eckart potential for triaxial nuclei. The European Physical Journal Plus, 131, 1-6

Suparmi. (2011). Mekanika Kuantum II. FMIPA UNS, Surakarta

Suparmi, A., Cari, C., \& Elviyanti, I.L. (2017). Solution of Klein Gordon equation for trigonometric cotangent potential in the presence of a minimal length using Asymtotic Iteration Method. IOP Conf. Series : Journal of Physics: Conf. Series 909012003 